\title{
AC-driven double quantum dots as spin pumps and spin filters.
}

\author{
Ernesto Cota ${ }^{1}$, Ramón Aguado ${ }^{2}$ and Gloria Platero ${ }^{2}$ \\ 1-Centro de Ciencias de la Materia Condensada - UNAM, Ensenada, Mexico. \\ 2-Instituto de Ciencia de Materiales, CSIC, Cantoblanco, Madrid,28049, Spain.
}

(Dated: October 25, 2018)

\begin{abstract}
We propose and analyze a new scheme of realizing both spin filtering and spin pumping by using acdriven double quantum dots in the Coulomb blockade regime. By calculating the current through the system in the sequential tunneling regime, we demonstrate that the spin polarization of the current can be controlled by tuning the parameters (amplitude and frequency) of the ac field. We also discuss spin relaxation and decoherence effects in the pumped current.

PACS numbers: $85.75 .-\mathrm{d}, 73.23 . \mathrm{Hk}, 73.63 . \mathrm{Kv}$
\end{abstract}

The emerging field of spintronics aims at creating devices based on the spin of electrons [1]. One of the most important requirements for any spin-based electronics is the ability to generate a spin current. Proposals for generating spin-polarized currents include spin injection by using ferromagnetic metals 2] or magnetic semiconductors [3]. Alternatively, one may use quantum dots (QDs) as spin filters or spin pumps [4, 5]. For QD spin filters, dc transport through few electron states is used to obtain spin-polarized currents as demonstrated experimentally by Hanson et al $\underline{6}$ following the proposal of Recher et al 7]. Spin current rectification has also been realized [8]. The basic principle of spin pumps is, on the other hand, closely related to that of charge pumps. In a charge pump a dc current is generated by combining ac driving with either absence of inversion symmetry in the device, or lack of time-reversal symmetry in the ac signal. The range of possible pumps includes turnstiles, adiabatic pumps or nonadiabatic pumps based on photon-assisted tunneling (PAT) [9, 10].

In this Letter we propose and analyze a new scheme of realizing both spin filtering and spin pumping by using a double quantum dot (DQD), in the Coulomb blockade regime, with time-dependent gate voltages and in the presence of a uniform magnetic field. The periodic variation of the gate potentials allows for a net dc current through the device even with no dc voltage applied [1, 12]: if the system is driven at a frequency (or subharmonic) corresponding to the energy difference between two time-independent eigenstates, the electrons become completely delocalized [13, 14]. If the left reservoir (chemical potential $\mu_{L}$ ) can donate electrons to the left dot (at a rate $\Gamma_{L}$ ) and the right reservoir (chemical potential $\mu_{R}$ ) can accept electrons from the right dot (at a rate $\Gamma_{R}$ ) the system will then pump electrons from left to right, even when there is no dc bias applied, namely $\mu_{L}=\mu_{R}$ (Fig. 1a). Starting from this pumping principle our device has two basic characteristics: $i$ ) If the process involves two-particle states, the pumped current can be completely spin-polarized even if the contact leads are not spin polarized and ii) the pumping can occur either through singlet (Fig. 1b) or triplet (Fig. 1c) states de- pending on the applied frequency, such that the degree of spin polarization can be tuned by means of the ac field. For example, if one drives the system (initially prepared in a state with $n=n_{L}+n_{R}=3$ electrons: $\left.|L=\downarrow \uparrow, R=\uparrow\rangle\right)$ at a frequency corresponding to the energy difference between the singlets in both dots, the electron with spin $\downarrow$ becomes delocalized in the DQD system. If now the chemical potential for taking $\downarrow(\uparrow)$ electrons out of the right dot is above (below) $\mu_{R}$, a spin-polarized current is generated. The above conditions for the chemical potentials can be achieved by breaking the spin-degeneracy through a Zeeman term $\Delta_{z}=g \mu_{B} B$, where $B$ is the external magnetic field (which is applied parallel to the sample in order to minimize orbital effects), $g$ is the effective g-factor and $\mu_{B}$ the Bohr magneton.

Our main findings can be summarized in Fig. 2 where we present a plot of the pumped current as a function of the applied frequency for a particular choice of parameters. Importantly, the current presents a series of peaks which are uniquely associated with a definite spin polarization: the pumped current is $100 \%$ spin-down (up) polarized for $\omega=\omega_{\downarrow}\left(\omega=\omega_{\uparrow}\right)$. The other peaks correspond to subharmonics of the energy difference between the relevant states, see below. This particular example illustrates how by tuning the external ac field one can operate the driven DQD as a bipolar spin filter with no dc voltage applied.

Formalism.- Our system consists of an asymmetric DQD connected to two reservoirs kept at the chemical potentials $\mu_{\alpha}, \alpha=L, R$. Using a standard tunneling Hamiltonian approach, we write for the full Hamiltonian $\mathcal{H}_{l}+\mathcal{H}_{D Q D}+\mathcal{H}_{T}$, where $\mathcal{H}_{l}=\sum_{\alpha} \sum_{k_{\alpha}, \sigma} \epsilon_{k_{\alpha}} c_{k_{\alpha} \sigma}^{\dagger} c_{k_{\alpha} \sigma}$ describes the leads and $\mathcal{H}_{D Q D}=\mathcal{H}_{Q D}^{L}+\mathcal{H}_{Q D}^{R}+\mathcal{H}_{L \Leftrightarrow R}$ describes the DQD. It is assumed that only one orbital in the left dot participates in the spin-polarized pumping process whereas two orbitals in the right dot (energy separation $\Delta \epsilon$ ) have to be considered. The isolated left dot is thus modelled as a one-level Anderson impurity: $\mathcal{H}_{Q D}^{L}=\sum_{\sigma} E_{L}^{\sigma} d_{L \sigma}^{\dagger} d_{L \sigma}+U_{L} n_{L \uparrow} n_{L \downarrow}$, whereas the isolated right dot is modelled as: $\mathcal{H}_{Q D}^{R}=\sum_{i \sigma} E_{R i}^{\sigma} d_{R i \sigma}^{\dagger} d_{R i \sigma}+$ $U_{R}\left(\sum_{i} n_{R i \uparrow} n_{R i \downarrow}+\sum_{\sigma, \sigma^{\prime}} n_{R 0 \sigma} n_{R 1 \sigma^{\prime}}\right)+J \mathbf{S}_{0} \mathbf{S}_{1}$. The index $i=0,1$ denotes the two levels. In practice, we take 

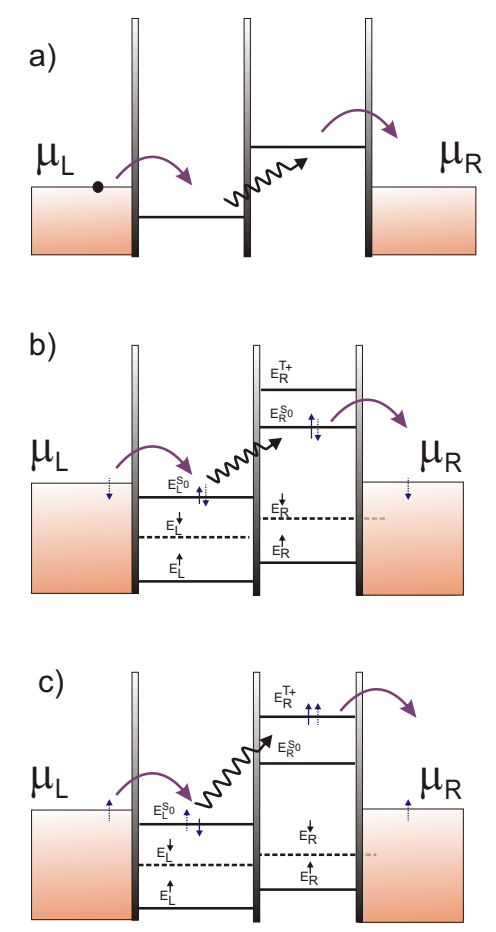

FIG. 1: (Color online) Schematic diagram of the double dot electron pump. a) Pumping through one-particle states: the current is spin-unpolarized. b) Pumping through two particle states with $\hbar \omega_{\downarrow}=E_{R}^{S_{0}}-E_{L}^{S_{0}}$ (where $E_{L}^{S_{0}}$ and $E_{R}^{S_{0}}$ are the energies of the singlets). If the chemical potentials fulfil the conditions $\mu_{1,0}(2,1)<\mu_{L}, \mu_{2,0}(1,2)>\mu_{R}$ and $\mu_{2,1}(1,2)<\mu_{R}$ (see text) the resulting pumped current is spin-down polarized. c) Pumping involving a triplet $\left(E_{R}^{T_{+}}\right)$in the right dot. In this case the pumped current is spin-up polarized. Note that during the pumping process only electrons with spin down (case b) or up (case c) become delocalized by the microwaves. Dashed arrows denote delocalized spins whereas solid arrows denote spins that remain localized on each dot.

$E_{L}^{\uparrow}=E_{R 0}^{\uparrow}=0\left(E_{L}^{\downarrow}=E_{R 0}^{\downarrow}=\Delta_{z}\right)$, so all the asymmetry is included in the charging energies $U_{R}>U_{L}$. Experimentally, this asymmetry can be realized by making the right dot smaller. $\mathbf{S}_{i}=(1 / 2) \sum_{\sigma \sigma^{\prime}} d_{R i \sigma}^{\dagger} \sigma_{\sigma \sigma^{\prime}} d_{R i \sigma^{\prime}}$ are the spins of the two levels. As a consequence of Hund's rule, the intra-dot exchange, $J$, is ferromagnetic $(J<0)$ such that the singlet $\left|S_{1}\right\rangle=(1 / \sqrt{2})\left(d_{R 0 \uparrow}^{\dagger} d_{R 1 \downarrow}^{\dagger}-d_{R 0 \downarrow}^{\dagger} d_{R 1 \uparrow}^{\dagger}\right)|0\rangle$ is higher in energy than the triplets $\left|T_{+}\right\rangle=d_{R 0 \uparrow}^{\dagger} d_{R 1 \uparrow}^{\dagger}|0\rangle$, $\left|T_{0}\right\rangle=(1 / \sqrt{2})\left(d_{R 0 \uparrow}^{\dagger} d_{R 1 \downarrow}^{\dagger}+d_{R 0 \downarrow}^{\dagger} d_{R 1 \uparrow}^{\dagger}\right)|0\rangle$ and $\left|T_{-}\right\rangle=$ $d_{R 0 \downarrow}^{\dagger} d_{R 1 \downarrow}^{\dagger}|0\rangle$. Due to the Zeeman splitting $E^{T_{-}}>E^{T_{0}}>$ $E^{T_{+}}=\Delta \epsilon+U_{R}-J / 4$. Finally, we consider the case where $\Delta \epsilon>\Delta_{z}+J / 4$ such that the triplet $\left|T_{+}\right\rangle$is higher in energy than the singlet $\left|S_{0}\right\rangle=(1 / \sqrt{2})\left(d_{R 0 \uparrow}^{\dagger} d_{R 0 \downarrow}^{\dagger}-\right.$ $\left.d_{R 0 \downarrow}^{\dagger} d_{R 0 \uparrow}^{\dagger}\right)|0\rangle . \quad \mathcal{H}_{L \Leftrightarrow R}=\sum_{i, \sigma} t_{L R}\left(d_{L \sigma}^{\dagger} d_{R i \sigma}+\right.$ h.c. $)$ describes tunneling between dots. The tunneling between leads and each QD is described by the perturbation $\mathcal{H}_{T}=$ $\sum_{k_{L}, \sigma} V_{L}\left(c_{k_{L} \sigma}^{\dagger} d_{L \sigma}+\right.$ h.c. $)+\sum_{i, k_{R}, \sigma} V_{R}\left(c_{k_{R} \sigma}^{\dagger} d_{R i \sigma}+\right.$ h.c. $)$. $\Gamma_{L, R}=2 \pi \mathcal{D}_{L, R}\left|V_{L, R}\right|^{2}$ are the tunneling rates. It is assumed that the density of states in both leads $\mathcal{D}_{L, R}$ and the tunneling couplings are energy-independent.

We study the system by a reduced density matrix (RDM), $\rho=\operatorname{Tr}_{L} \chi$, where $\chi$ is the full density matrix, and $\operatorname{Tr}_{L}$ is the trace over the leads. The dynamics of the RDM is formulated in terms of the eigenstates and eigenenergies of each isolated QD. We concentrate on the Coulomb blockade regime (with up to two electrons per dot, which defines a basis of 20 states) and study the sequential tunneling regime (Born-Markov approximation). For example, the diagonal elements of the RDM read

$$
\dot{\rho}_{s s}=-\frac{i}{\hbar}\left[\mathcal{H}_{L \Leftrightarrow R}, \rho\right]_{s s}+\sum_{m \neq s} W_{s m} \rho_{m m}-\sum_{k \neq s} W_{k s} \rho_{s s}
$$

where $W_{i j}$ are the transition rates (calculated using a standard Fermi Golden Rule). In addition we consider an ac field acting on the dots, such that the single particle energy levels become $\epsilon_{L(R)} \rightarrow \epsilon_{L(R)}(t)=$ $\epsilon_{L(R)} \pm \frac{e V_{A C}}{2} \cos \omega t$, where $e V_{A C}$ and $\omega$ are the amplitude and frequency, respectively, of the applied field. We include spin relaxation and decoherence phenomenologically in the corresponding elements of the equation for the RDM. Relaxation processes are described by the spin relaxation time $T_{1}=\left(W_{\uparrow \downarrow}+W_{\downarrow \uparrow}\right)^{-1}$, where $W_{\uparrow \downarrow}$ and $W_{\downarrow \uparrow}$ are spin-flip relaxation rates fulfilling a detailed balance. A lower bound for the spin relaxation time $T_{1}$ of $50 \mu \mathrm{s}$ with a field $B=7.5 T$ has been obtained recently [15] for a single electron in a QD using energy spectroscopy and relaxation measurements. In the following, we focus on zero temperature results such that $W_{\downarrow \uparrow}=0$ and thus $T_{1}=W_{\uparrow \downarrow}^{-1}$. The rate $T_{2}^{-1}\left(T_{2}\right.$ is the spin decoherence time) describes intrinsic spin decoherence. We take $T_{2}=0.1 T_{1}$ in all the calculations $[16$.

In practice, we integrate numerically the dynamics of the RDM in the chosen basis. In particular, all the results shown in the next paragraphs are obtained by letting the system evolve from the initial state $|\downarrow \uparrow, \uparrow\rangle$ until a stationary state is reached. The dynamical behavior of the system is governed by rates which depend on the electrochemical potentials of the corresponding transitions. The electrochemical potential $\mu_{1(2), i}\left(N_{1}, N_{2}\right)$ of $\operatorname{dot} L(R)$ is defined as the energy needed to add the $N_{1(2)}$ th electron to energy level $i$ of $\operatorname{dot} L(R)$, while having $N_{2(1)}$ electrons on $\operatorname{dot} R(L)$ 10. The current from left to right is: $I_{L \rightarrow R}(t)=\Gamma_{R} \sum_{s} \rho_{s s}(t)$, with a similar expression for $I_{R \rightarrow L}$. Here, states $|s\rangle$ are such that the right dot is occupied. For ease in the notation, we take from now on $\hbar=e=1$, such that $V_{A C}, \omega$, etc, have units of energy.

Results.- A calculation of the stationary current, for each direction of spin, namely $I_{t o t}=\sum_{\sigma=\uparrow, \downarrow} I_{\sigma}$ as a function of $\omega$ (and fixed intensity $V_{A C}=V_{A C}^{0}=0.7$ ), gives the results shown in Fig. 2. The main peak of $I_{\downarrow}$ (con- 


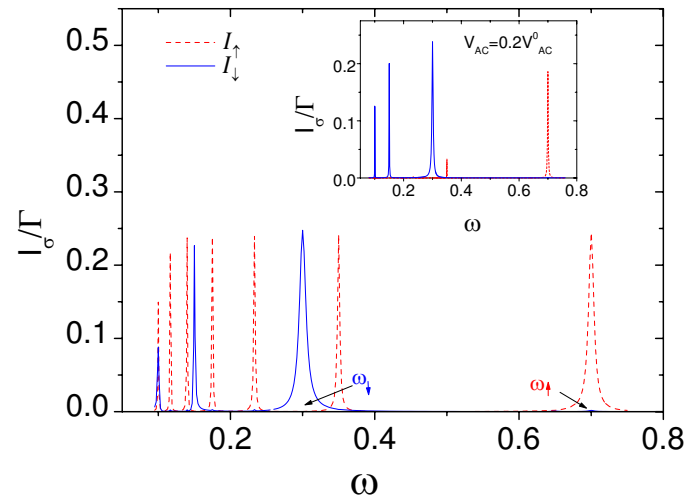

FIG. 2: (Color online) Pumped current as a function of the ac frequency. The spin-down component (solid line) shows three peaks corresponding to one $\left(\omega=\omega_{\downarrow}=0.3\right)$, two $(\omega=0.15)$ and three $(\omega=0.1)$ photon processes, respectively. The spin-up component (dashed line) shows a main one-photon resonance at $\omega=\omega_{\uparrow}=0.7$ and up to six more satellites corresponding to multiphoton processes. Parameters: $\Gamma_{L}=\Gamma_{R}=0.001, t=0.005, U_{L}=1.0, U_{R}=1.3, J=0.2$, $\mu_{L}=\mu_{R}=1.31, \Delta_{z}=0.026, \Delta \epsilon=0.45, V_{A C}=V_{A C}^{0}=0.7$ (in meV) correspond to typical values in GaAs QDs. In particular, the Zeeman splitting corresponds to a magnetic field $B \approx 1 T$. Inset: same as in main figure but with a lower intensity $V_{A C}=V_{A C}^{0} / 5=0.14$.

tinuous line) occurs at $\omega=\omega_{\downarrow} \equiv E_{(\uparrow, \downarrow \uparrow)}-E_{(\downarrow \uparrow, \uparrow)}=U_{R}-$ $U_{L}=0.3$ (see caption) At this frequency $I_{\uparrow} \approx 0$ (dashed line) demonstrating the efficiency of the spin-polarized pump. For this particular case, pumping of spin-down electrons occurs as one electron with spin $\downarrow$ becomes delocalized (via a one photon process) between both dots. If the chemical potential for taking $\downarrow$ electrons out of the right dot fulfils $\mu_{2,0}(1,2)=U_{R}-E_{\uparrow}>\mu_{R}$ while, on the other hand, the chemical potential for taking $\uparrow$ electrons out of the right dot fulfils $\mu_{2,1}(1,2)=U_{R}-E_{\downarrow}<\mu_{R}$ the resulting pumped current is spin-down polarized. We emphasize again that this pumping of spin polarized $(\downarrow)$ electrons is realized with unpolarized leads. Such spinpolarized current is obtained either through the sequence $(\downarrow \uparrow, \uparrow) \stackrel{\mathrm{AC}}{\Leftrightarrow}(\uparrow, \downarrow \uparrow) \stackrel{\Gamma_{R}}{\Rightarrow}(\uparrow, \uparrow) \stackrel{\Gamma_{\digamma}}{\Rightarrow}(\downarrow \uparrow, \uparrow)$ or, alternatively, $(\downarrow \uparrow, \uparrow) \stackrel{\mathrm{AC}}{\Leftrightarrow}(\uparrow, \downarrow \uparrow) \stackrel{\Gamma_{L}}{\Rightarrow}(\downarrow \uparrow, \downarrow \uparrow) \stackrel{\Gamma_{f}}{\Rightarrow}(\downarrow \uparrow, \uparrow)$. Reducing the frequency to $\omega=\omega_{\downarrow} / 2=0.15$, there is a second peak (corresponding to the absorption of two photons) in the spin down current. A three-photon process occurs at $\omega=\omega_{\downarrow} / 3=0.1$.

By increasing the frequency to $\omega=\omega_{\uparrow} \equiv E_{(\downarrow, \uparrow \uparrow)}-$ $E_{(\downarrow \uparrow, \uparrow)}=\Delta \epsilon+U_{R}-U_{L}-J / 4=0.7$ (see Fig. 2 ), a current peak with spin up polarization appears. In this case, the pumped current occurs as one electron with spin $\uparrow$ becomes delocalized between the states $(\downarrow \uparrow, \uparrow)$ and $(\downarrow, \uparrow \uparrow)$. This spin up electron subsequently decays to the right reservoir which leads to a pumped current through the sequence $(\downarrow \uparrow, \uparrow) \stackrel{\mathrm{AC}}{\Leftrightarrow}(\downarrow, \uparrow \uparrow) \stackrel{\Gamma_{R}}{\Rightarrow}(\downarrow, \uparrow) \stackrel{\Gamma_{\digamma}}{\Rightarrow}(\downarrow \uparrow, \uparrow)$ or $(\downarrow \uparrow$ $, \uparrow) \stackrel{\mathrm{AC}}{\Leftrightarrow}(\downarrow, \uparrow \uparrow) \stackrel{\Gamma_{\zeta}}{\Rightarrow}(\downarrow \uparrow, \uparrow \uparrow) \stackrel{\Gamma_{R}}{\Rightarrow}(\downarrow \uparrow, \uparrow)$. At $\omega_{\uparrow}, I_{\downarrow} \approx 0$ such that the spin polarization, defined as

$$
P\left(\omega, V_{A C}\right) \equiv \frac{I_{\uparrow}-I_{\downarrow}}{I_{\uparrow}+I_{\downarrow}},
$$

has been completely reversed by tuning the frequency of the ac field, namely $P\left(\omega_{\uparrow}, V_{A C}^{0}\right)=1=-P\left(\omega_{\downarrow}, V_{A C}^{0}\right)$. Note that the energy difference between both processes, $\omega_{\uparrow}-\omega_{\downarrow}=\Delta \epsilon-J / 4$ corresponds to the energy difference between the triplet excited state and the singlet ground state in the right dot at zero magnetic field.

Reducing the frequency to $\omega=\omega_{\uparrow} / 2$ and $\omega=\omega_{\uparrow} / 3$ and so on, peaks corresponding to absorption of up to seven photons appear. Note that each of these peaks has a different width. This remarkable fact can be attributed to a renormalization of the inter-dot hopping induced by the ac potential [9, 11]. At the frequency (or subharmonic) corresponding to the energy difference between two levels, the Rabi frequency becomes renormalized by the ac potential as $\Omega_{R a b i}=2 t_{L R} J_{N}\left(V_{A C} / \omega\right)$, $J_{N}$ is the Bessel function of order $N$ (=number of photons absorbed). The width of the peaks is given by the coupling to the leads provided that $\Gamma_{L, R}>\Omega_{R a b i}$. By contrast, if $\Gamma_{L, R}<\Omega_{R a b i}$ the width of the current peak is determined by $\Omega_{R a b i}$. Since $\Omega_{R a b i}$ depends on the number of photons in a nonlinear fashion (through the dependence of $J_{N}$ on the ratio $V_{A C} / \omega$ ), it follows that the widths of the peaks change in a non trivial way as a function of $\omega$. A similar nonlinear dependence of the height of the peaks as a function of the ratio $V_{A C} / \omega$ is expected. We illustrate this nontrivial behavior in the inset of Fig. 2 where we explore the low intensities regime $\left(V_{A C}=V_{A C}^{0} / 5=0.14\right)$. In general, the trend we obtain is consistent with previous analytical estimations 11].

Another interesting feature of the spin pump is that there are frequencies where the one-photon process corresponding to pumping of $\downarrow$ electrons can overlap with multiphoton processes corresponding to pumping of $\uparrow$ electrons. Thus, at these frequencies the current is no longer fully spin-polarized. One can use this to modify the polarization of the current by changing $V_{A C}$ (at fixed $\omega)$. We illustrate this with Fig. 3, where the parameters are chosen such that the $N=1$ peak of $I_{\downarrow}$ is centered at the same frequency $\left(\omega=\omega_{\downarrow}=0.3\right)$ as the $N=2$ peak of $I_{\uparrow}$. At this frequency, the spin polarization can be tuned by modifying the intensity of the ac potential (Fig.3, inset). This result, together with those shown in Fig. 2, demonstrate that the spin polarization of the current $P\left(\omega, V_{A C}\right)$ can be fully manipulated by tuning either the frequency or the intensity of the external ac field.

Finally, it is important to note also that, contrary to the case for spin-down pumping, the pumping of spin up electrons leaves the double dot in the excited state $|\downarrow, \uparrow\rangle$. This makes the spin-up current extremely sensitive to spin relaxation processes. If the spin $\downarrow$ decays 


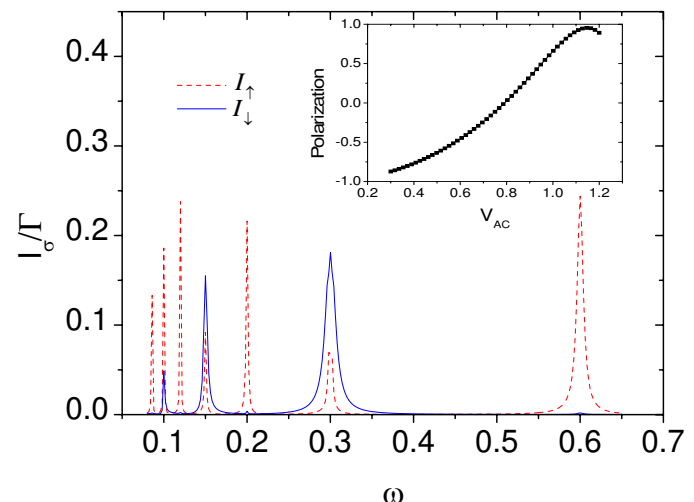

FIG. 3: (Color online) Pumped current as a function of the ac frequency. Same parameters as Fig. 2 except $\Delta \epsilon=0.35$. The interesting feature here is the overlap between the the onephoton absorption peak of $I_{\downarrow}$ (solid line) and the two-photon absorption peak of $I_{\uparrow}$ (dashed line) at $\omega=\omega_{\downarrow}=0.3$. The inset shows the spin polarization $P\left(\omega, V_{A C}\right)$ versus the ac intensity $V_{A C}$ for fixed frequency $\omega=\omega_{\downarrow}=0.3$ demonstrating the possibility of controlling the spin polarization of the current by tuning the intensity of the ac field.

before the next electron enters into the left dot, namely if $W_{\uparrow \downarrow} \gtrsim \Gamma_{L}$, a spin-down current appears through the cycle $(\downarrow \uparrow, \uparrow) \stackrel{\mathrm{AC}}{\Leftrightarrow}(\downarrow, \uparrow \uparrow) \stackrel{\Gamma_{R}}{\Rightarrow}(\downarrow, \uparrow) \stackrel{W_{\uparrow \downarrow}}{\Rightarrow}(\uparrow, \uparrow) \stackrel{\Gamma_{\digamma}}{\Rightarrow}(\uparrow \downarrow, \uparrow)$ and the pumping cycle is no longer $100 \%$ spin-up polarized. We study this effect in Fig. 4, where we plot the main PAT peak at $\omega_{\uparrow}=0.6$ for increasing $W_{\uparrow \downarrow}$. As one expects, the peak broadens as $W_{\uparrow \downarrow}$ increases. The full widths (FWHM) of the resonances are plotted as a function of $W_{\uparrow \downarrow}$ in the inset. For large intensities $\left(V_{A C}=V_{A C}^{0}\right.$, circles) the FWHMs grow in a nonlinear fashion. This is reminiscent of the so-called saturation regime, a well known phenomenon in the context of optical Bloch equations [17]. Note, however, that there are three energy scales involved now in the dynamics of the density matrix, $\Omega_{R a b i}, \Gamma$ and $W_{\uparrow \downarrow}$, such that other sources of nonlinearity (like the ones described when discussing Fig. 2) cannot be completely ruled out 18. In order to minimize nonlinear effects we investigate the low intensity regime $\left(V_{A C}=V_{A C}^{0} / 5\right.$, squares) where we expect a FWHM dominated by decoherence. The behavior is now linear with a slope which, interestingly, approaches FWHM $\sim 20 W_{\uparrow \downarrow}=2 / T_{2}$. Thus, experiments along these lines would complement the information about decoherence extracted from other setups [19].

Summary and experimental accessibility.-In summary, we have proposed and analyzed a new scheme of realizing both spin filtering and spin pumping by using ac-driven double quantum dots coupled to unpolarized leads. Our results demonstrate that the spin polarization of the current can be manipulated (including fully reversing) by just tuning the parameters of the ac field. Our results

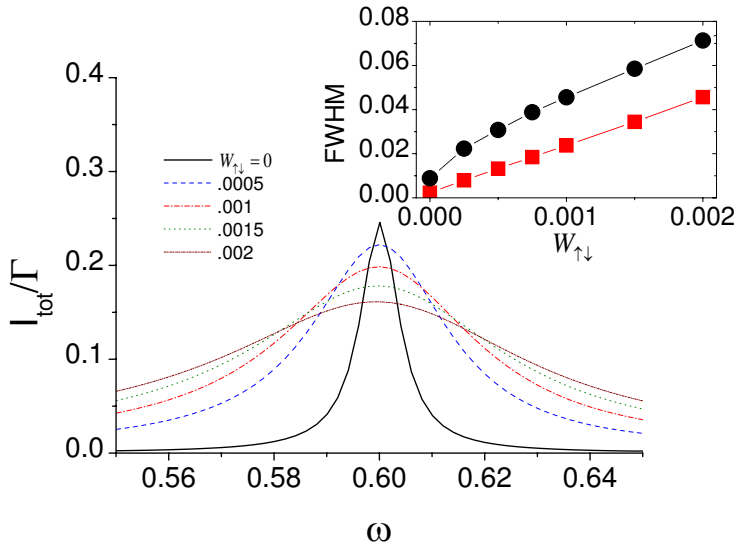

FIG. 4: (Color online) Pumped current near resonance $\omega=$ $\omega_{\uparrow}=0.6$ for different relaxation rates. Inset: FWHM of the total current as a function of relaxation rate, for strong (black dots) and weak (red squares) field intensity

also show that the width in frequency of the spin-up pumped current gives information about spin decoherence in the quantum dot. We finish by mentioning that our proposal is within reach with today's technology for high-frequency experiments in quantum dots 10, 13, 14]. Indeed, PAT with two-electron spin states has recently been reported [20].

We thank Wilfred van der Wiel for his help. Work supported by Programa de Cooperación Bilateral CSICCONACYT, by grant DGAPA-UNAM 114403-3 (E.C), by the EU grant HPRN-CT-2000-00144 and by the Ministerio de Ciencia y Tecnología of Spain through grant MAT2002-02465 (R. A. and G. P.) and the "Ramón y Cajal" program (R. A.).

[1] S. A. Wolf et al., Science 294, 1488 (2001).

[2] M. Johnson and R. H. Silsbee, Phys. Rev. Lett. 55, 1790 (1985); F. J. Jedema et al., Nature(London) 410, 345 (2001).

[3] R. Fiederling et al., Nature (London) 402, 787 (1999); Y. Ohno et al., Nature (London) 402, 790 (1999).

[4] E. R. Mucciolo et al., Phys. Rev. Lett. 89, 146802 (2002); Susan K. Watson et al., Phys. Rev. Lett. 91, 258301 (2003); M. G. Vavilov et al., cond-mat/0410042

[5] T. Aono, Phys. Rev. B 67, 155303 (2003); Qing-feng Sun et al., Phys. Rev. Lett. 90, 258301 (2003); E. Cota et al., Nanotechnology 14, 152-156 (2003).

[6] R. Hanson et al. Phys. Rev. B, 70, 241304 (2004).

[7] P. Recher et al. Phys. Rev. Lett., 85, 1962 (2000).

[8] K. Ono et al. Science 297, 1313 (2002); A. C. Johnson et al. cond-mat/0410679

[9] G. Platero and R. Aguado, Physics Reports, 395, 1 (2004).

[10] W. G. van der Wiel et al., 'Photon assisted tunneling in quantum dots', in: I.V. Lerner, et al. (Eds.), Strongly Correlated Fermions and Bosons in Low-dimensional 
Disordered Systems, Kluwer Academic Publishers, pp. 43-68 (2002).

[11] C. A. Stafford and N. S. Wingreen Phys. Rev. Lett. 76, 1916 (1996).

[12] B. L. Hazelzet, M. R. Wegewijs, T. H. Stoof, and Yu. V. Nazarov, Phys. Rev. B 63, 165313 (2001).

[13] T. H. Oosterkamp et al., Nature (London) 395, 873 (1998).

[14] J. R. Petta et al., cond-mat/0408139

[15] R. Hanson et al., Phys. Rev. Lett. 91, 196802 (2003).

[16] A detailed study of spin relaxation and decoherence in a GaAs quantum dot due to spin-orbit interaction can be found in, Vitaly N. Golovach et al., Phys. Rev. Lett. 93, 016601 (2004).

[17] Robert W. Boyd, Non-linear Optics (Academic Press, NY 2003)

[18] A detailed analytical study of the dynamics of the effective few level problem (to be published elsewhere) is needed in order to substantiate these arguments.

[19] See also, Hans-Andreas Engel and Daniel Loss, Phys. Rev. Lett., 86, 4648 (2001).

[20] T. Kodera, W. G. van der Wiel, K. Ono, S. Sasaki, T. Fujisawa, and S. Tarucha, Physica E, 22, 518 (2004). 